\title{
Das Labor für Teamarbeit und Moderation der TFH Wildau
}

\author{
Willi K. M. Dieterle
}

\section{Problemstellung}

Methoden der Teamarbeit und Moderation gehören seit vielen Jahren zu den am weitesten verbreiteten und populärsten partizipativen Arbeitsformen. Das ständig steigende Interesse an Teamentwicklungstrainings weist in diesem Zusammenhang darauf hin, das eine zunehmende Anzahl von Unternehmen die Art und Weise ihrer Zusammenarbeit optimieren und effizienter gestalten wollen. Hierbei ist die Moderationsmethodik von besonderer Bedeutung, weil sie eine Reihe kognitiver und affektiver Lernziele - wie im Folgenden im Einzelnen erläutert wird - gleichzeitig realisiert.

Darüber hinaus haben Lernzielanalysen ergeben, dass insbesondere Fachhochschulstudenten an einer verstärkten praxis- und arbeitsmarktorientierten Ausbildung interessiert sind[1]. Vor diesem Hintergrund einer verstärkten praxisorientierten hochschuldidaktischen Orientierung haben Hochschulleitung und Fachbereichsrat auf Antrag des Verfassers dieses Beitrags im Sommer 1999 beschlossen, das Labor Teamarbeit und Moderation an der TFH Wildau einzurichten.

\section{Voraussetzungen und Rahmenbedingungen}

Zu den Haupteinflussfaktoren effektiver Teamarbeit gehört insbesondere auch eine teamarbeitsgerechte Gestaltung der Lern- und Arbeitsumgebung. Die in diesem Zusammenhang relevanten Anforderungen sind bei der Laborgestaltung lediglich ansatzweise - vor allem aufgrund der bestehenden Raumknappheit - berücksichtigt worden, wie im Folgenden im Einzelnen erläutert wird.

\subsection{Raumbedarf}

Eine situationsgerechte Kalkulation des Raumbedarfs geht von einem Mittelwert von mindestens 5 qm pro Teilnehmer aus, d. h. bei einer Gruppengröße von 30 Teilnehmern müssten 150 qm zur Verfügung stehen[2]. Dieser erhöhte Raumbedarf resultiert aus spezifischen Anforderungen hinsichtlich Raumart und -zahl. Bei moderationsgerechten Arbeitssituationen hat sich im Sinne effizienter Prozessbedingungen ein differenziertes Vorgehen hinsichtlich Plenar- und Teamarbeit bewährt: Im Plenum, d. h. der Gesamtgruppe, erfolgen grundsätzliche Abstimmungen, Diskussionen und Präsentationen, die einzelnen Teamarbeitssituationen vor- bzw. nachgelagert sind. Anzahl und Größe der Teams werden in Abhängigkeit von den Besonderheiten des jeweiligen Problemlösungsprozesses gebildet. Diese Teams arbei- ten parallel an ausgewählten Teilaspekten der Problemstellung. Um dieses methodische Vorgehen realisieren zu können, muss der Raum entweder groß genug sein, um ihn teilen zu können, oder es müssen neben einem größeren Plenarraum mehrere kleinere Gruppenarbeitsräume zur Verfuigung stehen. Die aktuelle Gestaltung des Labors bietet derzeit nur einen mittelgroßen Plenarraum, der mit Hilfe von Stellwänden (inkl. Schallschutz) geteilt und von bis zu vier kleineren Teams gleichzeitig genutzt werden kann.

\subsection{Einrichtung/Moderationsmaterialien}

Aufgrund der spezifischen Anforderungen an moderationsgerechte Arbeitssituationen muss im Verhältnis von Raumgröße und Mobiliar ausreichend Bewegungsfreiheit fuir den Aktionsbereich eingeplant werden. Insofern ist bei der Einrichtung des Labors aufgrund der bestehenden Raumknappheit auf die Anschaffung von Interaktionstischen, die in der Regel zwischen den Teilnehmern platziert werden, verzichtet worden; diese Funktion wird vielmehr von Collegestuihlen mit integrierten Schreibflächen übernommen.

Zur Einrichtung des Labors gehören im Einzelnen:

- Moderatorenkoffer

Er enthält alle notwendigen Arbeits- und Verbrauchutensilien, die in der Moderationsarbeit eingesetzt werden.

- Regale

Sie dienen der Aufbewahrung weiterer Arbeitsmaterialien, insbesondere unterschiedlicher Kartenformen.

- Pinwände

Ihre entscheidenden Vorteile werden vor allem in den interaktionsorientierten Arbeits- und Visualisierungsflächen gesehen.

- ChartBoy

Er trägt bis zu 100 Blatt Pinwandpapier, ist rollbar und damit in unterschiedlichen Bereichen des Raumes einsetzbar.

- Magnetwand

Als zusätzliche Arbeitsfläche beispielsweise im Rahmen von Gruppenarbeit.

- Stellwände

Sie dienen als Raumteiler und sind ineinander verankerbar durch Klettverschlüsse. 


\section{- Farbtafeln}

Sie ermöglichen den Lernprozess unter dramaturgischen und farbpsychologischen Gesichtspunkten zu gestalten. Damit können spezifische Wirkungen in unterschiedlichen Lernsituationen erzielt werden.

- Collegestühle

Durch die integrierte Arbeitsplatte lassen sich raumsparend unterschiedliche Interaktionsformen zwischen Seminarteilnehmern realisieren. Ein weiterer Vorteil liegt in dem unterhalb der Sitzfläche befindlichen Ablagekorb, der mehrere Arbeitsmaterialien aufnehmen kann.

\section{- Schreibtisch}

Er dient als Einzelarbeitsplatz und bietet weitere Kommunikationsmöglichkeiten (PC und Telefon).

Mit dieser moderationsgerechten Ausstattung ist ein bedeutsamer Faktor erfolgreicher Teamarbeit gegeben. Hierbei stellt der Moderator geeignete Teamarbeitsmethoden und Techniken sowie sein Prozesswissen bzw. Prozesserfahrung zur Verfügung. Es werden vielfältige Gestaltungselemente, Kompositionsregeln und Darstellungsformen eingesetzt, insbesondere zur Textgestaltung mit Hilfe von Symbolen, Graphiken und Diagrammen sowie mit Hilfe von Farben[3].

\subsection{Technik/Elektronische Medien}

Derzeit stehen folgende Möglichkeiten zur Verfügung:

- PC-System

Für Datenrecherchen, Präsentationen etc.

- Daten- und Videoprojektorwand

Als weiteres Präsentationsmedium insbesondere im Rahmen von Plenararbeit.

- Videorecorder

Für die Präsentation von Videofilmen.

- Webcam

Für das PC-gestuitzte Fotografieren der Visualisate (Pinwände).

- CD-Player

Für den gezielten Einsatz von Musikproduktionen zur Erreichung eines spezifischen Lernklimas (Aktivierung, Entspannung etc.).

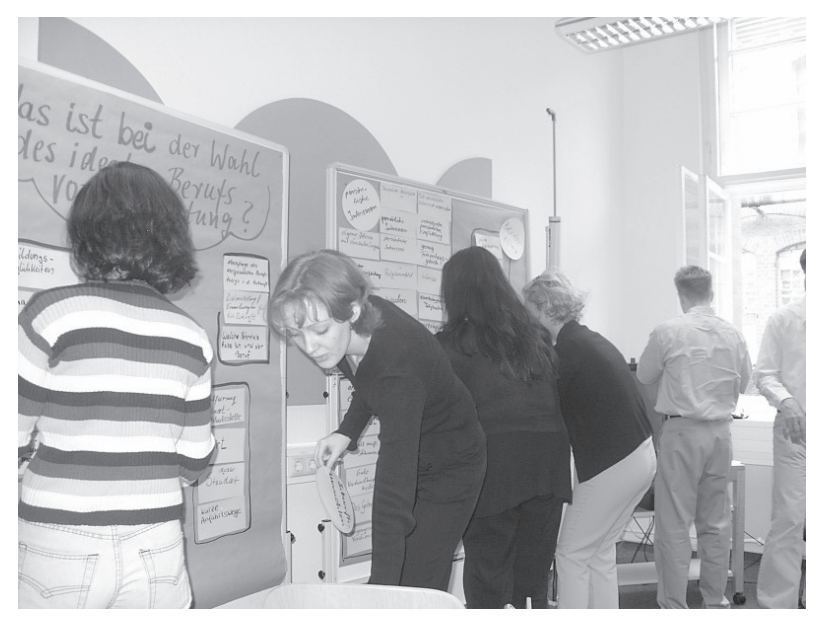

Abb. 1: Das Labor Teamarbeit und Moderation

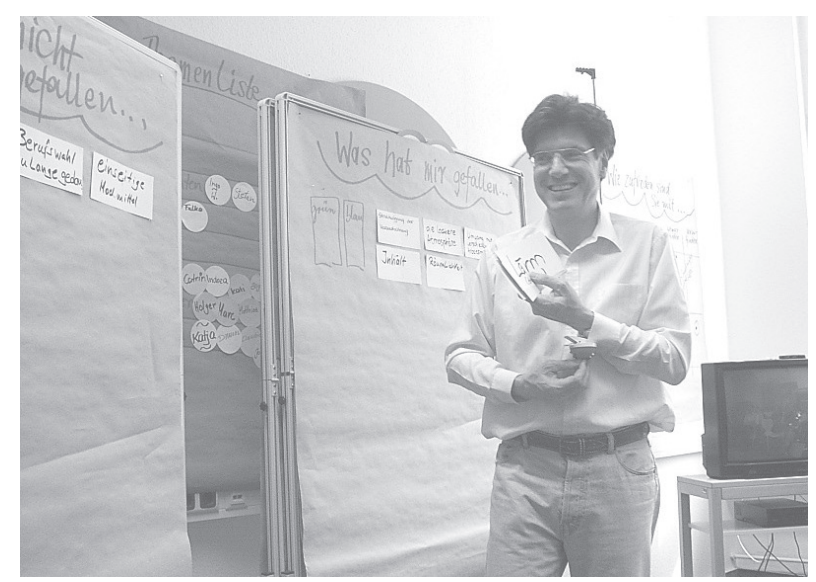

Abb. 2: Das Labor Teamarbeit und Moderation

\section{Anwendungsmöglichkeiten im Rahmen ausgewählter Lehrmethoden}

Im Folgenden werden Anwendungsmöglichkeiten im Rahmen hochschulverbreiteter Lehrmethoden aufgezeigt[4]:

\subsection{Lehrgespräch}

Die Visualisierung interessierender Diskussionsthemen (im Anschluss an die Vorträge/Referate) kann hier im Rahmen einer Zuruf-Abfrage realisiert werden. Die Themen werden in diesem Zusammenhang von dem Moderator (Lehrkraft) auf einer vorbereiteten Wandzeitung zügig aufgeschrieben (vgl. Abb. 3).

Durch diese Vorgehensweise werden Assoziationsketten ausgelöst, die eine umfassende Themensammlung gewährleisten. Im affektiven Bereich ist das verbesserte Lernklima hervorzuheben, das durch eine verstärkte Teilnehmerintegration gefördert wird (eine zu starke Beeinflussung durch „opinion leader“ kann vermieden werden).

Eine weitere Besonderheit liegt in dem veränderten Rollenverständnis, das von einer moderierenden Lehrkraft erworben werden muss: Während er sich als Moderator neutral verhält (reine Prozessbeteiligung), ist er als Lehrkraft Experte, der inhaltlich Stellung bezieht.

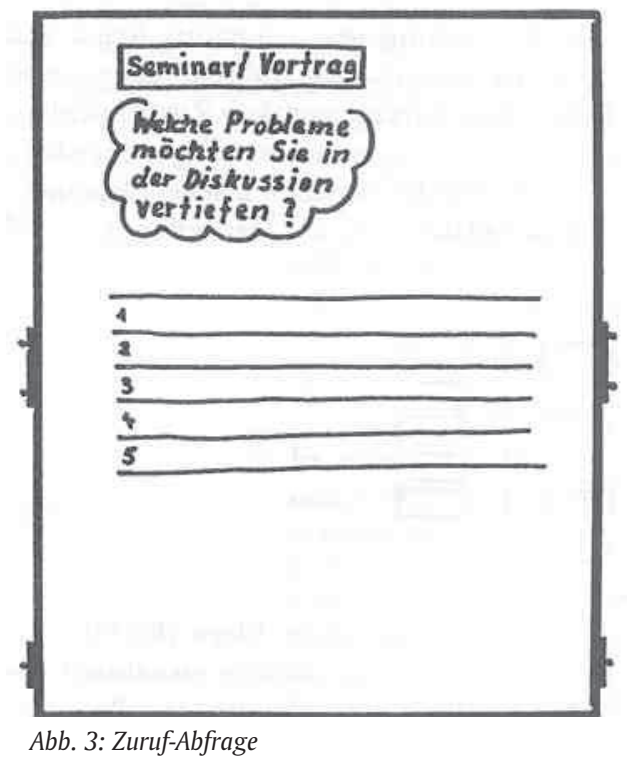




\subsection{Fallstudie}

Mit dem Einsatz der Fallmethode wird in der Regel das Ziel verbunden, den Lehrstoff auf konkrete Entscheidungssituationen zu übertragen, um dessen Gestaltungsrelevanz hinsichtlich der jeweiligen Problematik aufzuzeigen. Die Fallbearbeitung sollte sich hierbei an folgenden Lösungsschritten orientieren:

(a) Zielformulierung

Festlegung von Soll-Vorstellungen in möglichst operationaler Form

(b) Problemanalyse

(bl) Problemdefinition

Aufzeigen der Problemfelder und -symptome

(b2) Problemdiagnose

Formulierung von Hypothesen uiber die

Problemursachen (Ursachenanalyse)

(c) Problemlösung

(cl) Entwicklung von Lösungsvorschlägen (Alternativensuche)

(c2) Beurteilung konkurrierender Lösungsvorschläge hinsichtlich ihrer Eignung zur Erreichung der in (a) formulierten Ziele bzw. zur Handhabung der in (b) explizierten Probleme (Alternativenbewertung)

(c3) Erarbeitung eines Lösungsvorschlages (Auswahl und Begrüindung)

Für die Falldiskussion erscheinen alternierende Formen von Teamarbeit geeignet, die in parallelen Arbeitsgruppen stattfinden. Eine derartige Vorgehensweise kann die Realisierung sowohl kognitiver als auch affektiver Lernziele unterstuitzen. Hierzu gehören im Einzelnen:

- die Generierung einer möglichst großen und fundierten Anzahl von Lösungsalternativen,

- die Vermittlung eines stimulierenden Konkurrenzerlebnisses,

- die Fähigkeit zur Kooperation und Selbstorganisation in der Gruppe,

- die Fähigkeit zur Präsentation (Visualisierung) gemeinsam erarbeiteter Ergebnisse.

Die Integration von Gruppenarbeit leistet damit einen wirksamen Beitrag hinsichtlich einer sachlichen Kongruenz von Lern- und späteren Arbeitssituationen im Beruf.

In einer ersten Gruppenarbeitsrunde (4-5 Teilnehmer/ Gruppe, 1/2-stündig) können die Lösungsschritte Zielsetzung und Problemanalyse gemeinsam erarbeitet und eine entsprechende Präsentation vorbereitet werden. Hierbei empfiehlt sich der Einsatz der Moderations-Methodik: Über eine Kartenfrage können zunächst zu den o. g. Kategorien Vorschläge gesammelt, systematisiert und auf einer vorbereiteten Wandzeitung (Abb. 4) visualisiert werden.

Die Visualisate (Wandzeitungen als Ergebnis der Teamarbeit) können dann im Rahmen einer Plenarveranstal- tung präsentiert und diskutiert werden. Das Resultat der Plenardiskussion ist anschließend auf einer weiteren Wandzeitung als gemeinsamer Vorschlag festzuhalten.

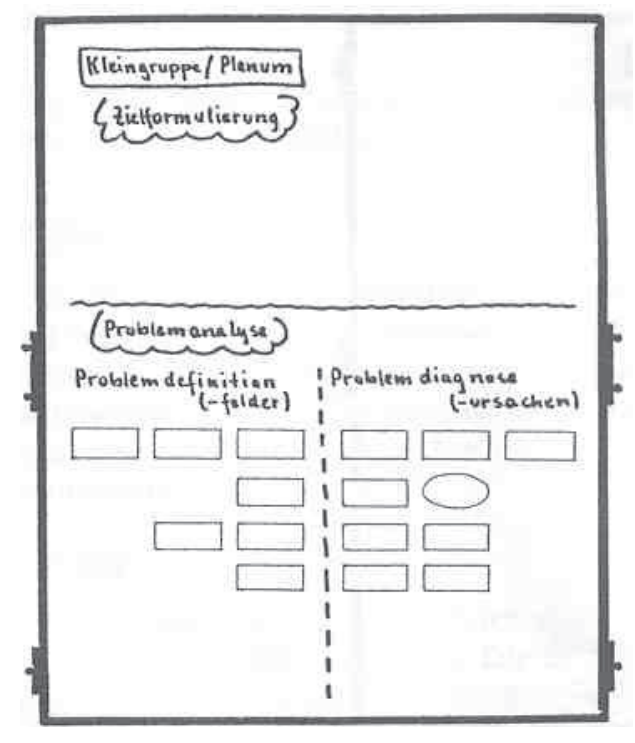

Abb. 4: Zielformulierung und Problemanalyse

Eine zweite Gruppenarbeitsrunde verfolgt schließlich das Ziel, eine fundierte Falllösung zu entwickeln. Hierbei könnten zunächst in einem ersten Schritt Lösungsmöglichkeiten im Hinblick auf theoretische Orientierungen unterschiedlicher Provenienz generiert werden (Abb. 5), während einem zweiten Schritt die Konzeptualisierung eines integrierten Lösungsvorschlages vorbehalten bliebe.

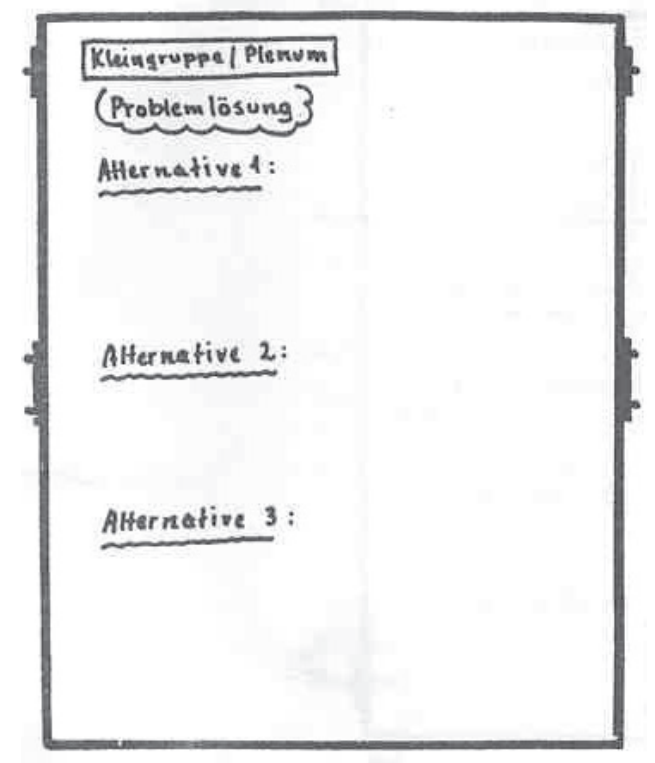

Abb. 5: Problemlösung - Alternativengenerierung

Darüber hinaus könnte eine Kategorie „Weitere Lösungsansätze“ vorgesehen werden, um den Teilnehmern die Möglichkeit zu eröffnen, außerhalb des konzeptionellen Rahmens liegende Vorschläge zu thematisieren bzw. die Kategorie „Worüber wir unbedingt noch sprechen müssen”, um offen gebliebene Fragen in inhaltlicher und/ oder methodischer Sicht für die anschließende Plenardiskussion festzuhalten (Abb. 6). 


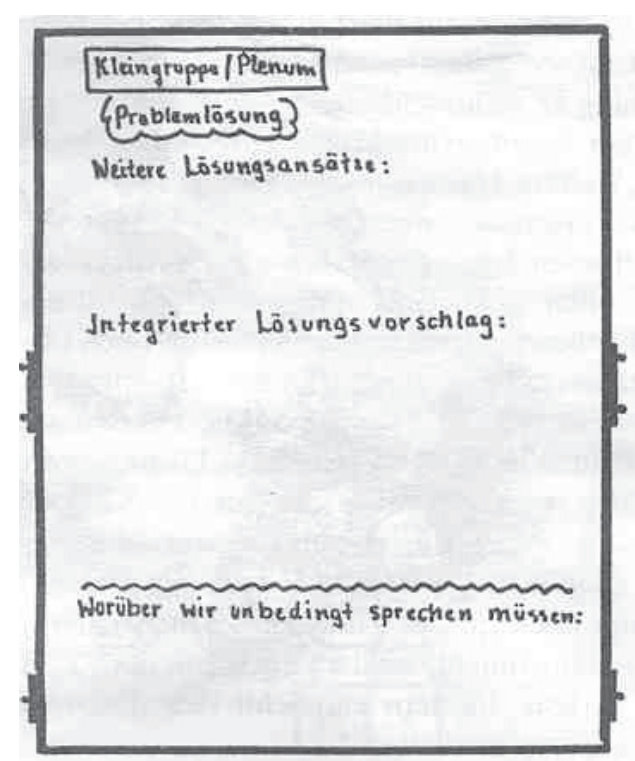

Abb. 6: Integrierte Problemlösung

In einer weiteren Plenarveranstaltung sollten dann die Visualisate der zweiten Teamarbeitsrunde präsentiert und diskutiert werden. Die Ergebnisse der Plenardiskussion sind schließlich im Hinblick auf die erarbeiteten Lösungsalternativen und den integrierten Lösungsvorschlag, der auch Elemente aus der Kategorie „Weitere Lösungsansätze“ beinhalten kann, auf entsprechend vorbereiteten Wandzeitungen festzuhalten. Darüber hinaus sollten offen gebliebene Fragen (Probleme) thematisiert werden (,Worüber wir unbedingt noch sprechen müssen“). Die diesbezüglichen Beiträge werden nicht nur von den Teilnehmern, sondern auch von der Lehrkraft formuliert (Stichwortsammlung auf Karten). Insofern empfiehlt sich deren teilnehmende Beobachtung in allen Phasen der Teamarbeit.

Eine falladäquate Ermittlung der Lehrzielerreichung könnte im Rahmen einer Selbstevaluierung der Gruppenmitglieder erfolgen. Als geeignete Frageform empfiehlt sich der Einsatz einer Mehrpunktfrage (vgl. Abb. 7).

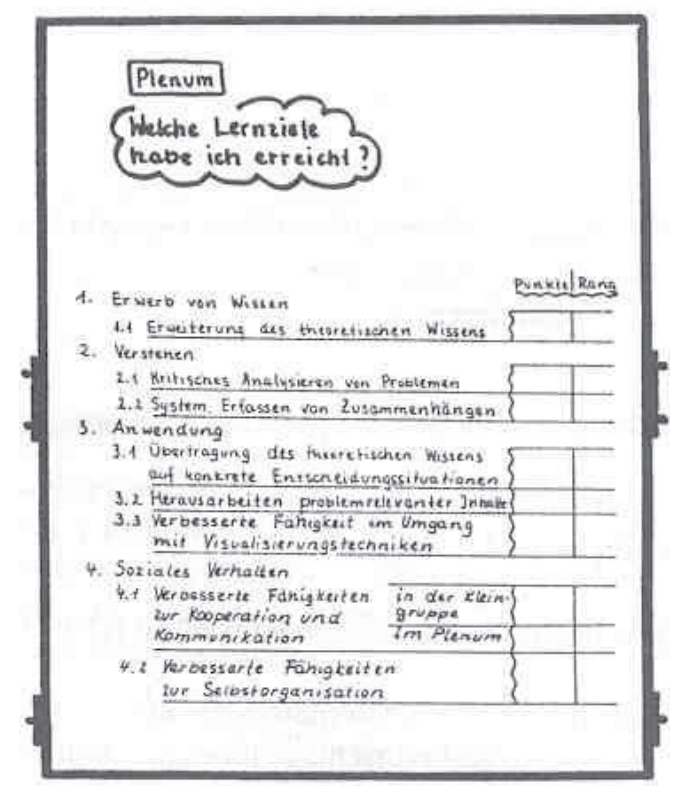

Abb. 7: Lehrzielkontrolle

\subsection{Rollenspiel}

Mit dem Einsatz dieser Lehrmethode wird das Ziel verbunden, die Teilnehmer zu befähigen, durch das „Spielen" vorgegebener Rollen (Berater/Management) ein tieferes Verständnis für unterschiedliche Positionen und Handlungsmuster zu erwerben. Ferner werden durch die Rollenübernahme Verhandlungsgeschick, Schlagfertigkeit und Überzeugungskraft entwickelt.

Ein derartig strukturiertes Rollenspiel könnte in der Weise vorbereitet werden, dass sich zunächst zwei Spielergruppen (zwei bis drei Teilnehmer je Gruppe) und eine Beobachtergruppe (Rest der Teilnehmer) mit der jeweiligen rollenspezifischen Problematik im Rahmen vorgeschalteter Teamarbeit eingehend auseinandersetzen. Eine Beurteilung des Lernprozesses könnte von der Beobachtergruppe mit Hilfe der Moderations-Methodik mediendidaktisch vorstrukturiert werden. Die Beurteilung erfolgt dann anhand geeigneter Kriterien (Abb. 8) im Rahmen einer verdeckten Kartenfrage (Abb. 9). In einer daran anschließenden Diskussion können Stärken und Schwächen einzelner Teilnehmer, die in den Verhaltenskategorien visualisiert werden, von allen Beteiligten im einzelnen erörtert werden.

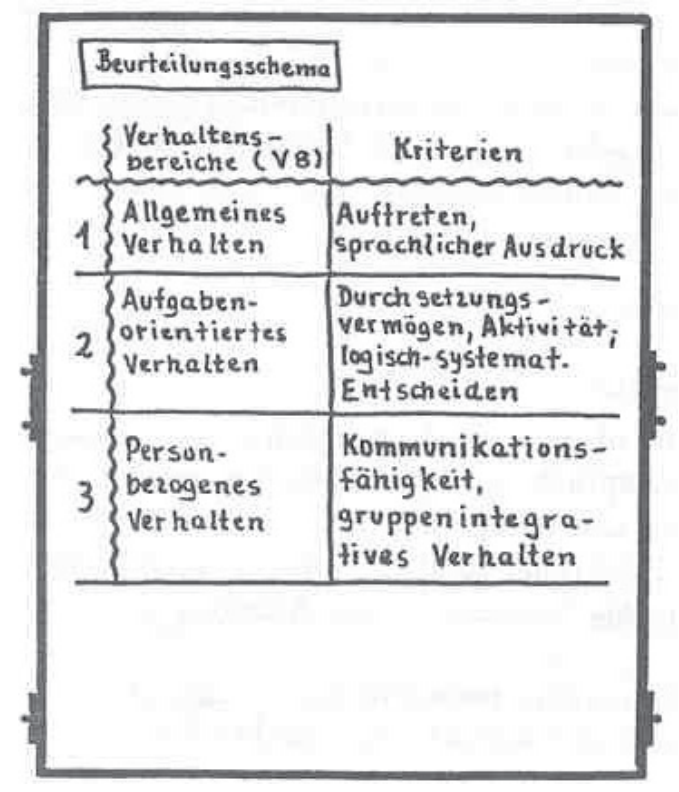

Abb. 8: Beurteilungskriterien (Rollenspiel)

\subsection{Projekt}

Seit Mitte der achtziger Jahre wird Handlungsorientierung als hochschuldidaktische Innovation in zunehmendem Maße diskutiert[5]. In diesem Zusammenhang wird u. a. auf die Projektmethode hingewiesen, die sich aufgrund von Befunden empirischer Erhebungen in der Business-Ausbildung und insbesondere in der Unternehmerausbildung (Entrepreneurship) bewährt hat[6]. Hierbei erscheint es zweckmäßig, wesentliche Teile der Ausbildung an Anforderungen auszurichten, die an die zukünftige Tätigkeit des Unternehmers gestellt werden. Diese sind im Einzelnen[7]:

- selbständige Zielausrichtung und Zielmodifikation,

- aktive und begrenzte Informationssuche, 


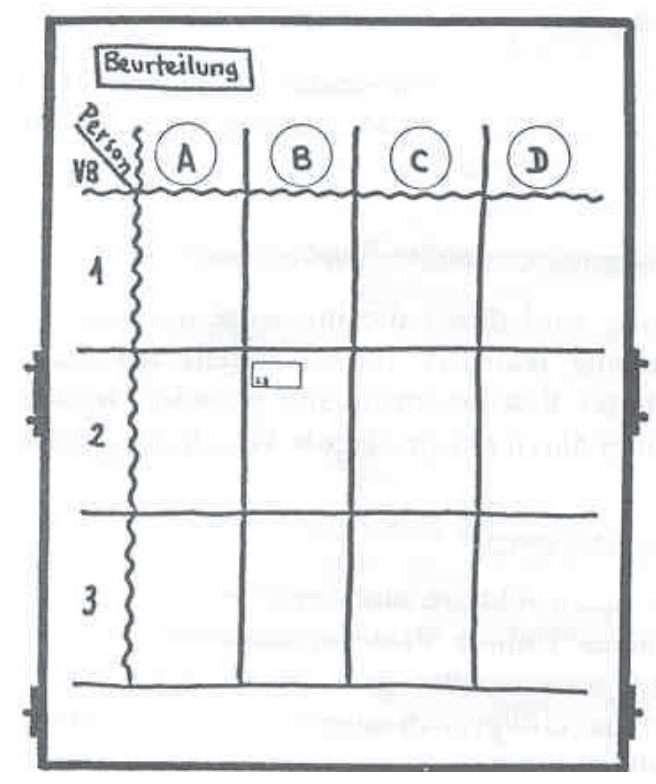

Abb. 9: Kartenfrage (Rollenspiel)

- analytische und ganzheitliche Problemdiagnose,

- entscheiden und handeln unter Zeitdruck.

Das Labor Teamarbeit und Moderation ermöglicht aufgrund der bestehenden Lern- und Arbeitsorganisation Projektarbeitssituationen, die den o. g. Anforderungen weitgehend gerecht wird.

\section{Didaktische/theoretische Implikationen moderationsgestützter Lehr-/Lernsituationen}

Im Folgenden sollen relevante Implikationen im Rahmen ausgewählter Dimensionen skizzenhaft umrissen werden[8].

\subsection{Mediendidaktik}

Die im Rahmen der Moderation angelegten medialen Repräsentationen des Unterrichtsgeschehens rechtfertigen die Charakterisierung derartiger Lehr-/Lernsituationen als medienorientiert, zumal der Medieneinsatz hier von eigenständiger, hervorgehobener Bedeutung ist. Die Effizienz bzw. die Besonderheiten eines derartigen Einsatzes können durch eine Reihe von Kriterien überprüft und angemessen dargestellt werden.

\section{(a) Steuerung}

Die Art der Steuerung ist hierbei durch eine nur teilweise Übertragung der Lehrfunktion auf Medien gekennzeichnet. Die Steuerungsrichtung ist in den einzelnen Phasen des Moderationsprozesses durchaus unterschiedlich: Phase der Informationssammlung: divergierend Problemanalyse: konvergierend Generierung von Lösungsalternativen: divergierend Beurteilung und Auswahl: konvergierend.

\section{(b) Individual-/Gruppenorientierung}

Moderationsorientierte Lehr-/Lernsituationen erfüllen dieses Kriterium sowohl auf der Gruppen- als auch auf der Individualebene. Die Gruppenorientierung wird durch die verstärkte Aktivierung aller Teilnehmer gewährleistet, so dass mit einer erhöhten Akzeptanz bzgl. der jeweiligen Ergebnisse gerechnet werden kann. Die Individualisierung des Lernprozesses wird einerseits in zeitlicher Hinsicht erreicht, indem beispielsweise im Rahmen von Kartenfragen nicht die Anzahl der zu ,produzierenden“ Karten vorgegeben wird, andererseits in inhaltlicher Hinsicht durch eine konsequente Einbeziehung von erfahrungsbezogenen Kenntnissen in den Lernprozess.

\section{(c) Objektivierung}

Die schrittweise Ablösung der darzustellenden Informationen von den Teilnehmern wird eine kritische Auseinandersetzung mit dem Lerngegenstand gefördert.

\section{(d) Anschaulichkeit}

Der Einsatz von Gestaltungsregeln und Darstellungsformen kann in jeder Phase des Lernprozesses ein hohes $\mathrm{Maß}$ an Anschaulichkeit gewährleisten.

\section{(e) Aktualität}

Im Rahmen von Moderationsprozessen wird der aktuelle Stand dokumentiert; Aktualität ist insofern zwangsläufig sichergestellt.

\section{(f) Wirtschaftlichkeit}

Die eingesetzten Medien sind im Vergleich zu traditionellen Technologien relativ kostenguinstig. Da auch deren Leistungen hinsichtlich der genannten Kriterien überzeugend sind, können sie im Sinne der intendierten Lernzielerreichung als wirtschaftlich bezeichnet werden.

\subsection{Lernpsychologie}

In neueren lernpsychologischen Untersuchungen wird die Frage erörtert, welche Mechanismen der Verankerung von Lerninhalten in den einzelnen Gedächtnisspeichern förderlich sind. Hierbei ist die Assoziationsbildung von entscheidender Bedeutung, d. h. die Aufnahme von Informationen ins Ultrakurzzeit- (UZG), Kurzzeit(KZG) und Langzeitgedächtnis (LZG) hängt von den vorhandenen Assoziationsmöglichkeiten ab (,innere Resonanz“). Diese wiederum können im Falle „mehrkanaligen“ Lernens optimal erweitert werden: Je mehr Eingangskanäle aktiviert werden, desto größer ist die Anzahl der entstehenden Gedankenverbindungen und deren Möglichkeit, in den jeweiligen Gedächtnisspeichern verankert zu werden. Dieser Sachverhalt wird in Abbildung 10 skizzenhaft umrissen.

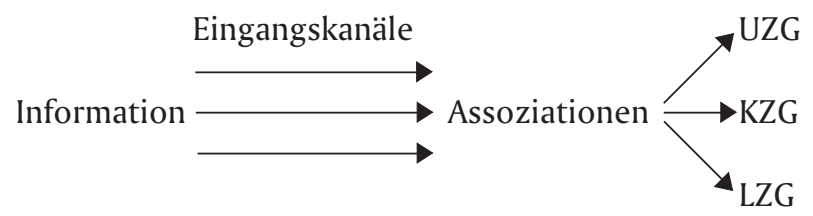

Abb. 10: Wahrnehmen und Behalten

Im folgenden soll untersucht werden, inwiefern Moderationsprozesse das Effizienzkriterium der Mehrkanaligkeit erfuillen. Hierbei wird die Unterscheidung in visuell, haptisch und auditiv zugrundegelegt. 


\section{(a) Visueller Eingangskanal}

Das permanente Visualisieren von Lernprozessen wird durch ein geeignetes didaktisches Instrumentarium gewährleistet.

\section{(b) Haptischer Eingangskanal}

Moderationsprozesse haben Werkstattcharakter, d. h. es erfolgt ein ständiger Umgang mit Materialien, um die Visualisate selbst herzustellen.

\section{(c) Auditiver Eingangskanal}

Durch die w. o. angesprochene didaktische Doppelfunktion moderierender Lehrkräfte erlangt die auditive Ebene eine neue Qualität.

Als Ergebnis kann festgehalten werden, dass moderationsorientierte Lehr-/Lernsituationen mehrkanalig angelegt sind und insofern verbesserte Übergänge in das Kurzzeit- bzw. Langzeitgedächtnis ermöglichen. Darüber hinaus können auch unterschiedliche Lerntypen wirksam in den Unterricht integriert werden.

\subsection{Humanistische Psychologie}

Humanistisch orientierte Didaktiken unterscheiden sich von traditionellen Konzeptionen vor allem durch deren Anspruch, eine ganzheitlich angelegte „Lernkultur” zu fördern, die insbesondere durch eine Integration kognitiver und affektiver Lernelemente bzw. eine zunehmende Erfahrungs- und Erlebnisbezogenheit der Lehr-/Lernsituationen gekennzeichnet ist. Als Vertreter dieser Richtung gelten u. a. Maslow, Rogers und Bühler.

Im Folgenden soll untersucht werden, inwiefern Moderationsprozesse in Erfuillung grundlegender Forderungen (Meta-Lernziele) diesen humanistischen Ansatz unterstiitzen.

\section{(a) Ganzheitliche Orientierung}

Eine ganzheitliche Orientierung wird im Sinne mehrkanalig angelegter Lernprozesse realisiert. Darüber hinaus werden affektive Lernelemente durch die verstärkte Integration aller Teilnehmer methodisch verankert.

\section{(b) Erhöhung der Lernselbständigkeit}

Der methodisch bedingte erweiterte Gestaltungsspielraum der Teilnehmer und deren Möglichkeit, Problemlösungsprozesse aktiv mitzugestalten, führen zu einer erhöhten Lernselbständigkeit.

\section{(c) Entwicklung einer intra- und interpersonellen Sensi- bilisierung}

Interpersonelle Sensibilisierung wird durch die intensive Auseinandersetzung mit den Auffassungen aller Teilnehmer realisiert. Intrapersonelle Sensibilisierung kann durch die Integration vorbewusster Bewusstseinsinhalte gefördert werden, die durch vermehrte Assoziationen bedingt durch entsprechende Visualisate - möglich wird.

\section{(d) Zunahme der sozialen Verantwortung}

Die Einbeziehung aller Beteiligten fuihrt zu einer erhöhten Akzeptanz der erarbeiteten Ergebnisse. Darüber hin- aus können Problemlösungsprozesse durch eine geeignete Methodik transparenter gemacht werden. Damit sind grundlegende Voraussetzungen für die erfolgversprechende Implementation gemeinsam erarbeiteter Entscheidungen erfiillt.

\subsection{Tätigkeitsfeldbezogene Aspekte}

In diesem Zusammenhang soll untersucht werden, welchen Beitrag moderationsorientierte Unterrichtsprozesse hinsichtlich einer sachlichen Kongruenz zwischen lern- und tätigkeitsfeldbezogenen Arbeitssituationen zu leisten imstande sind.

Wie bereits w. o. angedeutet wurde, können Moderationsprozesse im Sinne einer praxisorientierten Teamarbeitsmethodik interpretiert werden. Hierbei sind folgende Aspekte von Bedeutung:

- umfassendes Zusammenstellen problemrelevanter Informationen (,schriftliches Diskutieren“),

- Systematisieren durch Clusterbildung, Festlegen einer Rangordnung relevanter Themen,

- Problemanalyse, Problemlösung und Erarbeitung von Aktionsplänen in Arbeitskreisen.

In allen Phasen wird eine methodisch fundierte Unterstuitzung gewährt. Die Besonderheiten einer moderationsgestuitzten Teamarbeitsmethodik liegen in der Integration aller Beteiligten (verbesserte Implementationschancen und Termineinhaltung) und der mehrdimensional angelegten Kooperation (verbesserte Willensbildungsprozesse).

\section{Anwendungsfelder}

Im Folgenden sollen grundlegende Möglichkeiten der Nutzung des Labors Teamarbeit und Moderation uiberblicksartig aufgezeigt werden:

\section{- Seminaristische Nutzung}

Hierbei ermöglichen die unterschiedlichen Grundarbeitsformen Plenararbeit, Teamarbeit, Partnerarbeit und Einzelarbeit einen innovativen didaktischen Ansatz.

- Besprechung / Konferenz

Moderierte Besprechungen / Konferenzen fuihren zu verbesserten Ergebnissen.

\section{- Projektarbeit}

Eine „Werkstattatmosphäre“ führt zu funktionsgerechten, praxisnahen Projektarbeitssituationen, beispielsweise im Rahmen von Gründungsprojekten[9].

Die Belegung des Labors im Haus 13, Raum 123 erfolgt z. Z. über Frau Eileen Tschuschke (Stud. der Betriebswirtschaftslehre) in den jeweiligen Sprechstunden.

Der Autor dieses Beitrages möchte an dieser Stelle besonders Frau Peggy Brademann (Stud. der Betriebswirtschaftslehre) für die Unterstuitzung bei der Einrichtung 
sowie Herrn Dirk Rönnpagel (Stud. der Logistik und 1. Vorsitzender des City of Talents e.V.) für die Unterstiutzung bei der technischen Ausstattung des Labors danken.

\section{Anmerkungen}

[1] Vgl. iwd, Informationsdienst des Instituts der deutschen Wirtschaft, 18. Mai 2000, S. 2

[2] Vgl. Neuland, M.: Neuland-Moderation, Eichenzell 1995, S. 272

[3] Vgl. Dieterle, W. / Hinst, C.: Simultanvisualisierung als medienorientierte Lehr-/Lernsituation im betriebswirtschaftlichen Hochschulunterricht, in: Zeitschrift für Betriebswirtschaft, 1985, S. $383 \mathrm{ff}$.

[4] Vgl. ebenda, S. 389 ff.

[5] Vgl. Braukmann, U.: Förderung von Existenzgründungen aus Hochschulen - im Rahmen des bizeps-Projektes entwickelte Konturen einer Gründungsdidaktik, Wuppertal 2000, S. 25 ff.

[6] Vgl. Grüner, H.: Entrepreneurial Learning - Ist eine Ausbildung zum Unternehmertum möglich? In: Zeitschrift für Berufs- und Wirtschaftspädagogik, 1993, S. 496

[7] Vgl. Klandt, H.: Entrepreneurship spielend lernen. Erfahrungen beim Einsatz eines Computerplanspiels zur Vermittlung der mittelständischen Unternehmerrolle, in: Entrepreneurship. Wie aus Ideen Unternehmen werden, München 1998, S. 204

[8] Vgl. Dieterle, W. / Hinst, C., a.a.O., S. 395 ff.

[9] Initialisierung und Koordination erfolgt über den City of Talents e.V. an der Technischen Fachhochschule Wildau (www.city-of-talents.de)

\section{Autor}

Prof. Dr. Willi K. M. Dieterle

Technische Fachhochschule Wildau

Fachbereich Betriebswirtschaft/Wirtschaftsinformatik

Tel. (03375) 508-953

E-mail: dieterle@wi-bw.tfh-wildau.de 